\title{
Efectos graduales de la sonorización en las lenguas romances ${ }^{1}$
}

\author{
Jesús JiMÉnEZ ${ }^{1}$ y Maria-Rosa LLORET ${ }^{2}$ \\ ${ }^{1}$ Departamento de Filología Catalana, Universitat de València \\ ${ }^{2}$ Departamento de Filología Catalana, Universitat de Barcelona \\ jesus.jimenez@uv.es /mrosa.lloret@ub.edu
}

\begin{abstract}
RESUMEN
El objetivo de este trabajo es presentar los efectos sincrónicos de la asimilación de sonoridad en las lenguas romances y analizar los principios - las regularidades - que subyacen en la variación observada. Como la exégesis del fenómeno se verá condicionada por el dominio de aplicación, dentro de la palabra o entre palabras, y por su engranaje con los cambios que afectan a las obstruyentes en posición de coda y en posición de ataque, apuntamos en primer lugar las características comunes a los fenómenos de asimilación.
\end{abstract}

Palabras clave: asimilación, sonorización, cambio fonético.

[Recibido, septiembre 2013; aprobado, diciembre 2013]

Gradual effects of voicing

in the Romance languages

\begin{abstract}
The aim of this paper is to present the synchronic effects of voicing assimilation in Romance languages and analyze the principles - the regularities - that underlie the observed variation. As the exegesis of the phenomenon will be determined by the application domain within a word or between words and their gear with changes affecting obstructing in coda position and in attack, we aim, firstly, to show the common features of the assimilation phenomena.
\end{abstract}

Keywords: assimilation, voicing, phonetic change.

\footnotetext{
1 Este trabajo forma parte del proyecto de investigación FFI2013-46987-C3-1-P (www.ub.edu/GEVAD), financiado por el MINECO y el FEDER, y del grupo de investigación consolidado 2014SGR918, financiado por la Generalitat de Catalunya. Agradecemos a Aitor Carrera la ayuda que nos ha prestado con los datos del occitano.
} 


\section{Introducción}

El debate sobre la asimilación, en general, y sobre la asimilación de sonoridad, en particular, es sin duda ilustrativo de los intereses y de las preocupaciones de la fonología actual. Aunque se trata de un fenómeno caracterizado por una gran variabilidad, los patrones encontrados no son aleatorios sino que parecen obedecer a principios universales de la gramática, que ordenan y restringen las combinaciones de fenómenos posibles, lo cual encuentra un marco idóneo de formalización en la teoría de la optimidad (TO; Prince \& Smolensky 1993). En este caso, nos hallamos ante un proceso en el que confluyen, enfrentadas en un conflicto más o menos equilibrado, las dos fuerzas fundamentales de la gramática universal: la tendencia al mínimo esfuerzo articulatorio, en forma de principios de marcación, y la necesidad de mantener los contrastes para garantizar la comunicación, en forma de principios de fidelidad. Partiendo de estas premisas, el objetivo de este trabajo es presentar los efectos sincrónicos de la asimilación de sonoridad en las lenguas romances y analizar los principios - las regularidades - que subyacen en la variación observada. Como la exégesis del fenómeno se verá condicionada por el dominio de aplicación, dentro de la palabra (§3) o entre palabras $(\S 4)$, y por su engranaje con los cambios que afectan a las obstruyentes en posición de coda y en posición de ataque, apuntamos en primer lugar las características comunes a los fenómenos de asimilación $(\S 2)$.

\section{La asimilación como equilibrio inestable entre fidelidad y marcación}

La motivación principal que se aduce para justificar los procesos de asimilación es la simplificación articulatoria que suponen; así, la inercia de los articuladores puede provocar que un gesto articulatorio, como por ejemplo la vibración de las cuerdas vocales, se mantenga más allá del segmento al que se asocia originalmente. Esta fuerza motriz entra en conflicto con la posibilidad de que la extensión del rasgo asimilado comporte la neutralización superficial de especificaciones subyacentes. Por eso, la reducción gestual, formulada en TO en términos de restricciones de marcación, se ve limitada por las restricciones de fidelidad, que tratan de mantener los rasgos y los contrastes subyacentes. En esta coyuntura, el primer objetivo de este trabajo es analizar, a partir de la asimilación de sonoridad en las consonantes de las lenguas romances, las diferentes fórmulas que permiten equilibrar estas dos tendencias - normalmente - contradictorias.

Un segundo aspecto relacionado con la asimilación es la dirección en que se propagan los rasgos. Si solo se considerara la simplificación articulatoria, no deberían existir limitaciones sobre este aspecto: en principio, en una interacción entre dos unidades, obtendríamos el mismo beneficio sea cual sea el segmento cuyos rasgos se expandan, ya que en ambos casos reduciríamos de dos a una las especificaciones. Ahora bien, los datos indican que la dirección de la asimilación no es aleatoria, sino que tiene en cuenta las asimetrías perceptivas entre los segmentos. Un segundo objetivo del trabajo será mostrar la relevancia para los procesos de asimilación de la distinción entre segmentos asociados dentro de la palabra a posiciones prominentes, como el ataque silábico, el inicio de la palabra o, en general, el radical, y segmentos asociados a posiciones más débiles como la coda, el final de la palabra o los elementos clíticos. La inclusión de asimetrías diferentes de la relación entre ataques y codas en el tratamiento de la asimilación nos permitirá fundamentar uno de los puntos de las lenguas romances más conflictivos para un modelo paralelo de TO: la asimilación de sonoridad en consonantes pre- 
vocálicas a final de palabra, que, en lenguas como el catalán o el español de la Sierra del Ecuador, puede alterar un segmento resilabeado en una posición prosódicamente fuerte (i.e. el ataque silábico).

Finalmente, una tercera característica de la asimilación, que ha recibido menos atención en la bibliografía que las propiedades anteriores, es la correlación existente entre la semejanza de los segmentos y sus posibilidades de interacción. Así, si tomamos como referencia para definir la proximidad articulatoria entre dos segmentos su grado de constricción, es decir, su posición en la escala de sonoridad, se observa que cuanto más cercanos se encuentran dos elementos en esta escala, cuanto más similares son, más posibilidades existen de que uno se asimile al otro. Como resultado de esta correlación, los procesos de asimilación suelen presentar efectos graduales inclusivos, de manera que la existencia de interacción entre un segmento y otro elemento cualquiera de la escala suele implicar la posibilidad de que ambos segmentos también interactúen con otros elementos más próximos. Como veremos a lo largo del trabajo, este tipo de relaciones de implicación nos permitirá clasificar los patrones de variación en la asimilación de sonoridad de las lenguas romances según la permisividad que presenten respecto de la interacción entre las diferentes unidades.

\section{Sonorización en el ámbito de la palabra}

\subsection{Fidelidad en el ataque silábico}

En posición interior de palabra, las lenguas romances actuales preservan, de manera casi general, las especificaciones subyacentes de las obstruyentes prevocálicas (en $\S 3.3$, presentamos, sin embargo, algunos casos esporádicos de sonorización). En catalán, por ejemplo, los contrastes entre obstruyentes sordas y sonoras se mantienen regularmente delante de vocales (1). Por otra parte, las lenguas que, como el español, carecen de contraste en las fricativas y solo presentan variantes sordas conservan también dicha especificación ante vocal, incluso en la variedad de la Sierra del Ecuador, que, como veremos en $\S 4$, permite, igual que el catalán, la sonorización de algunas obstruyentes prevocálicas entre palabras (2).

(1) Catalán (estándar)

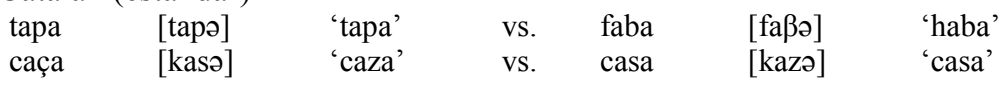

(2) Español (incluso en la variedad de la Sierra del Ecuador)



El mantenimiento de los rasgos de sonoridad en los ejemplos anteriores se atribuye en TO a una restricción de fidelidad referida especialmente al ataque silábico, IDENT $_{\mathrm{ON} \text { - }}$ ${ }_{\mathrm{SET}}(3){ }^{2}$ situada universalmente por encima de la restricción de fidelidad general IDENT (4)(cf. Lombardi 1995; 1996; 1999; Beckman 1998). La ordenación IDENT $_{\text {ONs }}>>$ IDENT se basa en la constatación de que los segmentos incorporados al ataque silábico ocupan una posición en que, gracias al notable contraste con el segmento siguiente - normal-

\footnotetext{
${ }^{2}$ Seguimos la convención habitual de mantener los nombres de las restricciones en inglés.
} 
mente, una vocal -, todos sus rasgos resultan especialmente perceptibles. Además, el principio IDENT $_{\mathrm{ONS}}$ dominaría en la jerarquía a la restricción de marcación $*[+$ voice, sonorant], que penaliza las obstruyentes sonoras en términos absolutos (5). Como mostramos en (6), esta jerarquía de principios garantiza la pervivencia de la especificación subyacente sonora de la sibilante intervocálica, a pesar de que este rasgo sea marcado. Cuando una consonante es subyacentemente sorda, como la fricativa de 0, IDENT $_{\text {ONs }}$ y * [+voice, - sonorant] actúan en la misma dirección y seleccionan el candidato con la variante fiel al input. No hace falta decir que los segmentos en posición de ataque inicial de palabra quedan protegidos igualmente por la restricción $\operatorname{IDENT}_{\mathrm{ONS}}$; siguiendo, e.g., con el ejemplo del catalán: /kasə/: [kasə], *[gasə] 'caza'; /gasə/: [gasə], *[kasə] 'gaza'.

(3) $\operatorname{IDENT}_{\mathrm{ONSET}}$ : El valor para $\left[{ }^{ \pm}\right.$sonoro] de un segmento del input coincide con el de su correspondiente en el output, si se silabea en el ataque.

(4) IDENT: El valor para [ \pm sonoro] de un elemento del input coincide con el de su correspondiente en el output.

(5) $*[+$ voice, - sonorant $]$ : Las obstruyentes son sordas.

(6) $/ \mathrm{kazə} /$ 'casa' (catalán)

\begin{tabular}{|c|l|l|l|}
\hline$/ \mathrm{kaz \partial} /$ & IDENT $_{\mathrm{ONS}}$ & $*[+$ voice, - sonorant $]$ & IDENT \\
\hline a. kasə & $* !$ & & $*$ \\
\hline b. kazə & & $*$ & \\
\hline
\end{tabular}

(7) $/ \mathrm{kas} ə /$ 'caza' (catalán)

\begin{tabular}{|l|l|l|l|}
\hline$/$ kasə/ & IDENT $_{\text {ONS }}$ & $*[+$ voice,- sonorant $]$ & IDENT \\
\hline a. kasə & & & \\
\hline b. kazə & $* !$ & $*$ & $*$ \\
\hline
\end{tabular}

\subsection{Marcación contextual y fidelidad en grupos de consonantes}

Los ejemplos del apartado anterior indican que, en ausencia de restricciones a favor de la asimilación, el principio IDENT $_{\mathrm{ONS}}$ obliga a mantener las especificaciones subyacentes de los segmentos asociados al ataque silábico; por tanto, los valores para [ \pm sonoro] de dichos segmentos dependen de su especificación léxica y son, básicamente, impredecibles. En cambio, cuando una obstruyente $(\mathrm{Ob})$ silabeada como coda precede a otra consonante situada en el ataque silábico, encontramos muy a menudo asimilación del primer segmento al rasgo [ \pm sonoro] de la segunda consonante. En este contexto, el catalán y el occitano (8) presentan un cuadro de asimilaciones bastante simple: todas las obstruyentes de la coda, sean oclusivas (T) o fricativas y africadas (S), toman el rasgo de sonoridad del segmento del ataque siguiente: una oclusiva, una fricativa o africada o una consonante sonante $(\mathrm{N})$. (En los ejemplos de este apartado, presen-

\footnotetext{
3 Algunas lenguas romances experimentan neutralización de sonoridad de las obstruyentes en posición final de palabra; así, en catalán (y en occitano), todas se muestran sordas: $s a[\mathrm{k}]$ 'saco', go [s] 'perro' (cf. $s a[\mathrm{k}] a$ 'saca', go[s]a 'perra'); ce[k] 'ciego', pi[s] 'piso' (cf. ce[y]a 'ciega', pi[z]os 'pisos'). Otras permiten el contraste entre obstruyentes sordas y sonoras; por ej., el francés: rose [воz] 'rosa' vs. rosse [воs] 'rocín', o el rumano: $d r a[\mathrm{k}]$ 'diablo' vs. $d r a[\mathrm{~g}]$ 'querido'. Aunque no formalizaremos esta cuestión aquí, la neutralización se sigue directamente de la ordenación $*[+$ voice, - sonorant $]>>$ IDENT propuesta para (6) y 0; para dar cuenta del mantenimiento del contraste, habría que suponer el orden inverso.
} 
tamos entre paréntesis realizaciones que comportan cambios adicionales no relacionados con el rasgo $[ \pm$ sonoro].)

(8) a. Catalán (estándar)

\begin{tabular}{|c|c|c|c|}
\hline TT: & ruptura & {$[\mathrm{pt}]$} & $\begin{array}{l}\text { 'ruptura' } \\
\text { 'madeja' }\end{array}$ \\
\hline & cabdell & & 'madeja' \\
\hline TS: & absolut & [ps] & 'absoluto' \\
\hline & objecte & [b3] & 'objeto' \\
\hline ST: & vista & [st] & 'vista' \\
\hline & desdiu & [zð] & 'desdice' \\
\hline TN & tècnica & {$[\mathrm{gn}]([\mathrm{gn}])$} & 'técnica' \\
\hline V: & $\begin{array}{l}\text { admirar } \\
\text { asma }\end{array}$ & $\begin{array}{l}{[\mathrm{dm}]([\mathrm{mm}])} \\
{[\mathrm{zm}]}\end{array}$ & $\begin{array}{l}\text { 'admirar' } \\
\text { 'asma' }\end{array}$ \\
\hline
\end{tabular}

b. Occitano (languedociano; Alibèrt 2000)

$\begin{array}{llll}\mathrm{TT}: & \text { acte } & {[\mathrm{tt}]} & \text { 'acto' } \\ & \text { cabdèl } & ([\mathrm{tt}] /[\mathrm{t}]) & \text { 'madeja' } \\ \mathrm{TS}: & \text { occitan } & {[\mathrm{ts}]} & \text { 'occitano' } \\ \mathrm{ST}: & \text { castigat } & {[\mathrm{st}]} & \text { 'castigado' } \\ & \text { esglasiar } & {[\mathrm{zY}]([\mathrm{y}])} & \text { 'asustar' } \\ \mathrm{TN}: & \text { atlèta } & ([11]) & \text { 'atleta' } \\ & \text { admetre } & ([\mathrm{mm}]) & \text { 'admitir' } \\ \mathrm{SN}: & \text { espasme } & {[\mathrm{zm}]} & \text { 'espasmo' }\end{array}$

En español, en gallego y en portugués, solo las fricativas se asimilan regularmente a las consonantes obstruyentes o sonantes del ataque siguiente; las consonantes oclusivas - que a menudo se ven afectadas por otros fenómenos - pueden mantener su especificación subyacente (9). En italiano, las oclusivas que no son fruto de geminadas solo aparecen en posición de coda preconsonática excepcionalmente, en préstamos o palabras cultas, por lo que la falta de concordancia en ejemplos como Etna en (10) es también poco significativa; la fricativa /s/, por su parte, se asimila al rasgo de sonoridad de la consonante siguiente. Finalmente, el rumano y el francés tienden a igualar los grupos de obstruyentes, pero admiten grupos de obstruyente y sonante discordantes en sonoridad (11).

(9) a. Español (estándar)

$\begin{array}{lllll}\text { TT: } & \text { ruptura } & ([\beta \mathrm{t}] & \text { abdicar } & {[\beta \delta]} \\ \text { TS: } & \text { absoluto } & ([\beta \mathrm{s}]) & & \\ \text { ST: } & \text { vista } & {[\mathrm{st}]} & \text { desde } & {[\mathrm{z}]} \\ \text { TN: } & \text { técnica } & {[\mathrm{kn}]} & \text { admirar } & {[ð \mathrm{~m}]} \\ \text { SN: } & \text { mismo } & {[\mathrm{zm}]} & & \end{array}$

b. Gallego (Freixeiro 2006)

$\begin{array}{llll}\text { TT: } & \begin{array}{l}\text { apto } \\ \text { abdicar }\end{array} & {[\mathrm{pt}]([\mathrm{bt}] /[\mathrm{t}])} & \text { 'apto' } \\ \mathrm{TS}: & \text { absoluto } & {[\mathrm{bs}]([\mathrm{s}] /[\mathrm{ws}])} & \text { 'abdicar' } \\ \mathrm{ST}: & \text { cuspir } & {[\mathrm{sp}]} & \text { 'essoluto' } \\ & \text { esvarar } & {[\mathrm{z}]} & \text { 'resbalar' } \\ \mathrm{TN}: & \text { técnica } & {[\mathrm{kn}]} & \text { 'técnica' } \\ & \text { admiración } & {[\mathrm{dm}]([ð \mathrm{dm}] /[\text { ðem }])} & \\ \mathrm{SN}: & \text { prosma } & {[\mathrm{zm}]} & \text { 'pachorra' }\end{array}$


c. Portugués (europeo; Mateus \& d'Andrade 2000)

\begin{tabular}{|c|c|c|c|}
\hline $\mathrm{T}^{\mathrm{T}}$ & adquirir & {$[\mathrm{dk}]$} & 'adquirir' \\
\hline & abdómen & [bd] & 'abdomen' \\
\hline TS: & absurdo & [bs] & 'absurdo' \\
\hline & abjurar & [b3] & 'abjurar' \\
\hline ST: & espaço & {$\left[\int \mathrm{p}\right]$} & 'espacio' \\
\hline & esbirro & [3b] & 'policía' \\
\hline TN: & étnico & {$[\mathrm{tn}]$} & 'étnico' \\
\hline & admirar & [dm] & 'admirar' \\
\hline SN & eslavo & {$[31]$} & 'eslavo' \\
\hline
\end{tabular}

(10) Italiano (Lombardía; Krämer 2009)

$\begin{array}{llll}\text { TT: } & \text { copto } & {[\mathrm{pt}]([\mathrm{tt}])} & \text { 'copto' } \\ \mathrm{ST}: & \text { vispo } & {[\mathrm{sp}]} & \text { 'vivaz' } \\ & \text { Fassbinder } & {[\mathrm{zb}]} & \\ \mathrm{TN}: & \text { Etna } & {[\mathrm{tn}]([\mathrm{nn}])} & \\ \mathrm{SN}: & \text { cosmo } & {[\mathrm{zm}]} & \text { 'cosmos' }\end{array}$

(11)a. Rumano (Cazacu et al. 1967; Wetzels \& Mascaró 2001)

\begin{tabular}{|c|c|c|c|}
\hline TT: & lapte & {$[\mathrm{pt}]$} & 'leche', \\
\hline & totdeauna & [dd] & 'siempre' \\
\hline TS: & absolut & [ps] & 'absoluto' \\
\hline & abject & [b3] & 'abyecto' \\
\hline ST & discuta & [sk] & 'discutir' \\
\hline & dezgust & {$[\mathrm{zg}]$} & 'disgusto' \\
\hline TN: & etnic & [tn] & 'étnico' \\
\hline & grabnic & [bn] & 'rápido' \\
\hline SN & basme & {$[\mathrm{sm}]$} & 'historia' \\
\hline & gleznă & [zn] & 'tobillo' \\
\hline
\end{tabular}

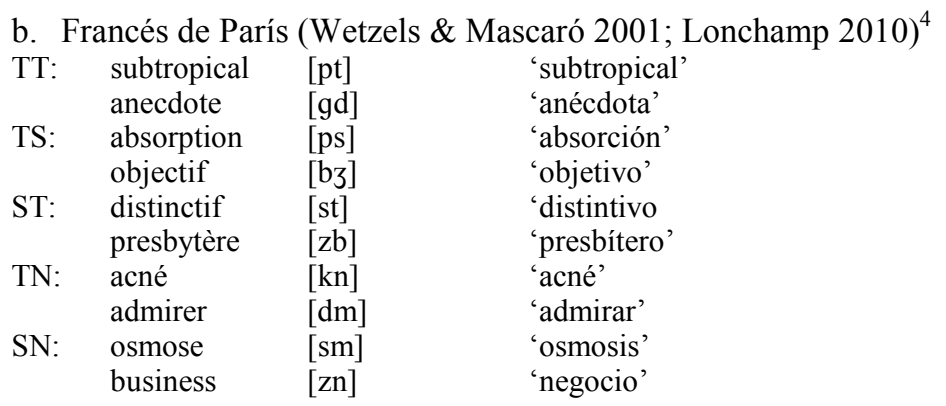

Al margen del comportamiento peculiar de las oclusivas preconsonánticas en español, gallego, italiano y portugués, que no trataremos aquí, observamos que las obstruyentes de la coda o bien se asimilan a cualquier consonante del ataque (en catalán, espa-

\footnotetext{
4 En los grupos de obstruyentes del francés, algunos autores, como Lonchamp (2010), presentan asimilados todos los encuentros entre obstruyentes, tanto en interior de palabra como entre palabras, en grupos primarios y secundarios. Otros autores distinguen entre asimilación obligatoria para el caso del ensordecimiento y opcional para el caso de la sonorización (Dell 1995, apud Wetzels \& Mascaró 2001). Finalmente, autores como Walter (1977) detectan, en conjunto, una cierta opcionalidad en ambos casos. En cualquier caso, la diferencia no es contrastiva.
} 
ñol, gallego, italiano, occitano y portugués), o bien se asimilan solo a otras obstruyentes (en rumano y francés). Esta división de los contextos asimilatorios se fundamenta en la semblanza articulatoria entre los segmentos implicados (cf., para propuestas similares, Itôet al. 1995; Rose \& Walker 2004; y, con relación a la asimilación de sonoridad en catalán, Jiménez 1999; Bermúdez-Otero 2001; Jiménez \& Lloret 2008). Las secuencias de consonantes obstruyentes, en palabras como desde, constituyen el contexto típico en que opera la exigencia de que la especificación para $[ \pm$ sonoro $]$ sea idéntica. Por una parte, dada la proximidad articulatoria entre los dos segmentos, es difícil realizarlos con una diferencia de sonoridad que sea al mismo tiempo perceptible. Por otra parte, estos segmentos se reparten entre dos sílabas, de manera que presentan normalmente una asimetría de prominencia que acentúa los rasgos del ataque y, al mismo tiempo, debilita los rasgos asociados a la posición más débil, esto es, la coda. Ambos factores influirían conjuntamente a favor de la amalgama segmental, una tendencia que recoge la restricción de (12).

(12) AGREE[ \pm voice $]_{\mathrm{ObOb}}$ : Los grupos de obstruyentes deben coincidir en el valor para [ ${ }^{ \pm}$sonoro] (cf. Lombardi 1996; 1999; Beckman 1998).

Las secuencias heterosilábicas de una obstruyente seguida de una consonante sonante, en palabras como mismo, comparten con los grupos de obstruyentes la estructura silábica. Sin embargo, articulatoriamente son suficientemente diferentes para que la tendencia asimilatoria sea menor. Sea como sea, se trataría de una asimilación de segundo orden respecto del caso tipológicamente más frecuente; en términos de principios, la restricción en contra de especificaciones disonantes en grupos de obstruyentes estaría universalmente por encima de la restricción de (13), que penaliza la falta de asimilación de una obstruyente a una sonante. ${ }^{5}$

(13) AGREE[ $[ \pm \text { voice }]_{\mathrm{ObN}}$ : Una obstruyente y la consonante sonante que la sigue deben coincidir en el valor para $[ \pm$ Sonoro $]$.

Por último, las obstruyentes prevocálicas constituirían el contexto menos proclive a la asimilación, puesto que en este caso encontramos la máxima distancia articulatoria entre los elementos y, además, las consonantes se silabean como ataques, de manera que todos sus rasgos son perfectamente perceptibles. El principio de (14), referido a la concordancia entre obstruyentes y vocales, ocuparía, por lo tanto, el escalón inferior de la jerarquía (15).

(14) AGREE[ \pm voice $]_{\mathrm{Obv}}$ : Una obstruyente y la vocal que la sigue deben coincidir en el valor para $[ \pm$ sonoro].

(15) Jerarquía: AGREE $[ \pm \text { voice }]_{\mathrm{ObOb}} \gg>$ AGREE $[ \pm \text { voice }]_{\mathrm{ObN}} \gg>$ AGREE $[ \pm \text { voice }]_{\mathrm{ObV}}$

El cuadro de (17) muestra cómo la incorporación de estos principios a la jerarquía permite seleccionar el resultado adecuado para la palabra mismo del español (consideramos, por congruencia con el sistema, que la sibilante es sorda subyacentemente). El candidato (17)a es descartado porque viola AGREE[ \pm voice $]_{\mathrm{ObN}}$, ya que las dos consonan-

5 Wheeler (2005) también modifica la restricción de (12) para incluir las obstruyentes ante sonantes, pero añadiendo la especificación de que las obstruyentes han de estar en la coda. 
tes contiguas tienen rasgos diferentes para $[ \pm$ sonoro]. La igualación vía ensordecimiento de la nasal en (17)c modifica los rasgos de un segmento asociado al ataque y, además, presenta una sonante sorda, una opción marcada que va contra la restricción universal *[ - voice, +sonorant](16). El candidato ganador es (17)b, que satisface AGREE $[ \pm \text { voice }]_{\mathrm{ObN}}$ con el coste mínimo para la jerarquía de principios.

$(16) *[-$ voice, + sonorant $]$ : Las sonantes son sonoras.

(17)/mismo/ (español)

\begin{tabular}{|c|c|c|c|c|c|}
\hline $\mathrm{mi} / \mathrm{sm} / \mathrm{o}$ & IDENT $_{\mathrm{ONS}}$ & AGREE $[ \pm \text { voice }]_{\mathrm{ObN}}$ & $*[-\mathrm{vc},+\mathrm{son}]$ & $*[+\mathrm{vc},-\mathrm{son}]$ & IDENT \\
\hline $\mathrm{a} . \mathrm{sm}$ & & $* !$ & & & \\
\hline$\approx \mathrm{b} . \mathrm{zm}$ & & & & $*$ & $*$ \\
\hline c. $\mathrm{sm}_{\odot}$ & $* !$ & & $*$ & & $*$ \\
\hline
\end{tabular}

Para evitar que haya asimilación en el contacto heterosilábico de obstruyentes y consonantes sonantes (como en rumano y francés en (11)), habría que suponer que la restricción AGREE[ $[ \pm \text { voice }]_{\mathrm{ObN}}$ se sitúa por debajo de *[+voice, - sonorant] y de IDENT en la jerarquía, de manera que estos grupos se mantengan sin asimilarse, como se muestra en 0 . En cambio, los grupos de obstruyentes en palabras como subtropical se asimilarían, por la presión de AGREE[ \pm voice $]_{\mathrm{ObOb}}$, como muestra el cuadro de 0 .

(18) osmose (francés)

\begin{tabular}{|c|c|c|c|c|c|c|}
\hline o/sm/ose & AGREE $[ \pm \mathrm{vc}]_{\mathrm{ObOb}}$ & IDENT $_{\mathrm{ONS}}$ & $*[-\mathrm{vc},+\mathrm{son}]$ & $*[+\mathrm{vc},-\mathrm{son}]$ & IDENT & AGREE[ $\pm \mathrm{Vc}]_{\mathrm{ObN}}$ \\
\hline a. $\mathrm{sm}$ & & & & & & $*$ \\
\hline b. $\mathrm{zm}$ & & & & $* !$ & $*$ & \\
\hline
\end{tabular}

(19) subtropical (francés)

\begin{tabular}{|c|c|c|c|c|c|c|}
\hline $\mathrm{sub} / \mathrm{bt} / \mathrm{ropical}$ & AGREE $[ \pm \mathrm{vc}]_{\mathrm{ObOb}}$ & IDENT $_{\mathrm{ONS}}$ & $\begin{array}{c}*[-\mathrm{vc}, \\
+\mathrm{son}]\end{array}$ & $*[+\mathrm{vc},-\mathrm{son}]$ & IDENT & AGREE $[ \pm \mathrm{vc}]_{\mathrm{ObN}}$ \\
\hline $\mathrm{a} . \mathrm{bt}$ & $* !$ & & & $*$ & & \\
\hline $\mathrm{b} . \mathrm{pt}$ & & & & & $*$ & \\
\hline
\end{tabular}

Alternativamente, para dar cuenta de la falta de asimilación de las obstruyentes a las sonantes en ejemplos como el de 0 , se ha apelado, en el marco de la teoría de la subespecificación, al carácter redundante $-\mathrm{y}$, por tanto, supuestamente inactivo - del rasgo [ ${ }^{\text {sonoro] }}$ en las sonantes (e.g., Kiparsky 1985; Archangeli 1988). ${ }^{6}$ Sin embargo, estudios actuales han demostrado su carácter activo en los procesos que presentan algunas lenguas (cf. Botma 2011). Por ejemplo, en español y en otras lenguas romances las sonantes provocan la asimilación de sonoridad de las obstruyentes, por lo que se necesita que el rasgo [+sonoro] esté activo en estos segmentos (cf. (17)). Además, el recurso a la subespecificación es problemático en TO porque se supone que, sean cuales sean las

\footnotetext{
${ }^{6}$ Dejamos de lado aquí la discusión sobre caracterizaciones alternativas de las sonantes (como mediante el rasgo [sonoridad espontánea], propuesto, entre otros, por Rice \& Avery 1989; 1991; Rice 1993), porque se han mostrado problemáticas; cf. Botma (2011).
} 
especificaciones subyacentes de los elementos, con rasgos activos o con rasgos pasivos, una jerarquía de principios adecuada debe ser capaz de derivar los resultados finales de manera correcta. Por ello, es más apropiado atribuir la posible falta de concierto sonoro en rumano y en francés a condiciones específicas referidas a la interacción entre las obstruyentes y las sonantes, como la que hemos presentado aquí (o como las que sugeriremos para la interacción entre obstruyentes y vocales en las secuencias postléxicas del punto 4.4).

Volviendo a la restricción AGREE $[ \pm \text { voice }]_{\mathrm{ObN}}$, la formulación que hemos presentado en (13) no hace referencia a las posiciones silábicas de la obstruyente y de la sonante. Por lo tanto, las obstruyentes habrían de tender a concordar con la sonante siguiente también cuando ambos elementos forman un ataque silábico complejo, como en la palabra plano del español, que analizamos en (20). Sin embargo, las potenciales igualaciones del rasgo [ \pm sonoro] en el grupo consonántico de (20)b y de (20)c se realizan alterando los rasgos de consonantes asociadas al ataque y, en el segundo caso, ensordeciendo además una sonante, por lo que es seleccionado el candidato (20)a, con valores discordantes para el rasgo [ \pm sonoro].

\section{(20)/plano/ (español)}

\begin{tabular}{|c|c|c|c|c|c|}
\hline$/ \mathrm{pla} / \mathrm{no}$ & IDENT $_{\mathrm{ONS}}$ & AGREE $[ \pm \text { voice }]_{\mathrm{ObN}}$ & $*[-\mathrm{vc},+\mathrm{son}]$ & $*[+\mathrm{vc},-\mathrm{son}]$ & IDENT \\
\hline a. pla & & $*$ & & & \\
\hline b. bla & $* !$ & & $*$ & $*$ & $*$ \\
\hline c. pla & $* !$ & & $*$ & & $*$ \\
\hline
\end{tabular}

En conjunto, pues, la principal limitación a la uniformización de los rasgos en los grupos consonánticos proviene de la necesidad de mantener las especificaciones de las consonantes asociadas al ataque, es decir, de la presión del principio IDENT $_{\text {ONS }}$. En este sentido, el hecho de que lenguas como el francés (21)a o el rumano (21)b, con contraste sordo vs. sonoro en toda la serie obstruyente, puedan mantener el rasgo sonoro original de las dos consonantes en algunos ataques complejos iniciales de palabra demuestra, de nuevo, que las posiciones prosódicas fuertes, como el ataque silábico inicial de palabra, son muy reticentes a los cambios, aun a costa de presentar discordancias de sonoridad en los grupos consonánticos que acogen. ${ }^{8}$

\footnotetext{
${ }^{7}$ El francés presenta alófonos total o parcialmente sordos de cualquier sonante en posición final absoluta (sable -[bl] 'arena', il part -[6] 'él parte',peuple -[pl] 'pueblo', rythme -[tmo] 'ritmo', rhumatisme -[sm] 'reumatismo') y en general tras obstruyentes sordas en el ataque de la sílaba (plan [pl]- 'plan', pneu [pn]- 'neumático', poids [pw]- 'peso', la semaine -[sm]- 'la semana') (Walter 1977: 35-36; Walker 1984: 36). En estas variedades, AGREE[ $[ \pm \text { voice }]_{\mathrm{ObN}}$ tiene en la jerarquía un impacto mayor y, por el contrario, *[ - voice, +sonorant], menor. Por otra parte, para atenuar el papel de IDENT $_{\mathrm{ONS}}$, se tiene que reducir su dominio a la posición inicial de los ataques - complejos o simples. De esa manera, se pueden modificar los rasgos de los elementos secundarios, bien porque están en la coda, bien porque son el segundo miembro de un ataque complejo. (Sobre la unificación prosódica de las codas y de los segundos miembros de una obertura compleja, v. Baertsch 2002 y Baertsch \& Davis 2003; sobre el papel de la prominencia posicional a la hora de decidir la dirección de la asimilación en ataques complejos secundarios, cf. §4.3.)

${ }^{8}$ En rumano, los ataques iniciales formados por /s/ seguida de oclusiva siempre concuerdan en sonoridad: spera [sp]- 'esperar', zbura [zb]- 'volar'. Del mismo modo, en italiano /s/ preconsonántica en ataque inicial de palabra toma el rasgo de la consonante siguiente: spugna [sp]- 'esponja', sbattere [zb]- 'sacudir' y también slanciare [zl]- 'echar hacia delante' (Krämer 2009: 209).
} 


$\begin{array}{lll}\text { (21)a. } & \begin{array}{l}\text { Francés (Durand 1992) } \\ \text { spire }\end{array} & \\ \text { sbire } & {[\mathrm{sp}]-} & \text { 'espiral' } \\ \text { sphère } & {[\mathrm{sb}]-} & \text { 'esbirro' } \\ \text { svelte } & {[\mathrm{sf}]-} & \text { 'esfera' } \\ \text { pneu } & {[\mathrm{pn}]-} & \text { 'esbelto' } \\ \text { gnose } & {[\mathrm{gn}]-} & \text { 'neumático' }\end{array}$

b. Rumano (Wetzels \& Mascaró 2001)

$\begin{array}{lll}\text { snoavă } & {[\mathrm{sn}]-} & \text { 'anécdota' } \\ \text { zmeură } & {[\mathrm{zm}]-} & \text { 'frambuesa' } \\ \text { cneaz } & {[\mathrm{kn}]-} & \text { 'príncipe' } \\ \text { gnostic } & {[\mathrm{gn}]-} & \text { 'agnóstico' }\end{array}$

\subsection{Redefiniendo los equilibrios: cambios esporádicos y estabilización}

El rasgo de sonoridad propio de las vocales también tiende a extenderse a las obstruyentes sordas en posición prevocálica e intervocálica, en virtud del principio AGREE[ \pm voice $]_{\mathrm{ObV}}$ de (14). Como estas consonantes se asocian al ataque silábico, deberían estar protegidas por la restricción de fidelidad IDENT $_{\mathrm{ONS}}$. Con todo, la sonorización, sobre todo en posición intervocálica, aparece esporádicamente en algunas lenguas romances y más a menudo en otras. ${ }^{9}$ En la Figura 1 ilustramos este fenómeno con la sonorización de la /p/ inicial de París en la secuencia Menja ceba a París [meņ̃̄za seßa baris] 'come cebolla en París' en un hablante del valenciano meridional.



Figura 1. Sonorización de $p$ intervocálica en la frase Menja ceba a París.

\footnotetext{
${ }^{9}$ No descartamos que, además de la restricción AGREE $[ \pm \text { voice }]_{\mathrm{ObV}}$, pueda actuar un principio independiente a favor de la sonorización de consonantes intervocálicas, como el que Krämer (2009: 40) propone en su análisis del italiano: «*VsV: Obstruents between vowels are voiced». De hecho, algunos autores sostienen que el contexto relevante para la sonorización ante sonante es la vocal precedente, por lo que la asimilación se interpreta como progresiva (v. Dyckstra 1955; Jansen 2004; Strycharczuk \& Simon 2013). En el apartado 4.2, sin embargo, presentamos ejemplos del catalán y del esp. del Ecuador que muestran que, sea cual sea el origen de la sonorización, sincrónicamente se debe atribuir a la vocal de la derecha de la obstruyente asimilada.
} 
En catalán, asimilaciones de este tipo son solo ocasionales. En cambio, la sonorización espontánea de obstruyentes, especialmente entre vocales, es común en muchas hablas hispánicas, como se indica en distintos puntos de la $N G L E(\S \S 4.5 \mathrm{~d}, 4.5 \mathrm{e}, 5.5 \mathrm{n}$, 5.9c). Por ejemplo, existen casos bastante sistemáticos de sonorización de $/ \mathrm{s} / \mathrm{y} / \theta /$, incluso tras consonante sonante, en la zona centro peninsular (Salamanca, Cáceres, Ávila, Badajoz y Toledo: huesos[gwezos], pues sí [pwezi], o sea [ozea], a ver si [aßerzi], manso [manzo];Torreblanca 1976; 1983; 1986). Otras veces, la sonorización de $s$ entre vocales aparece lexicalizada en palabras concretas, como en localidades de La Gomera y La Palma (Catalán 1964: 240-241, apud Lipski 1989: 151, n.1). La sonorización de oclusivas intervocálicas se documenta también en el español peninsular (Hualdeet al. 2011), es bastante general en hablas de las islas Canarias (Almeida Suárez 1982; Oftedal 1985) y variable en algunos hablantes de La Habana (Guitart 1980) (sobre esta cuestión, v. también Martín Butragueño en prensa).

A la larga, estos fenómenos de sonorización pueden consolidarse - convencionalizarse-, de manera que las variantes sonoras se conviertan en la realización habitual de las obstruyentes sordas intervocálicas, con o sin neutralización del contraste con las obstruyentes sonoras correspondientes (sobre la convencionalización de procesos fonéticos esporádicos, v. Hualde 2011; 2013). Así, la sonorización de oclusivas intervocálicas, con mantenimiento parcial del contraste, caracteriza a algunos dialectos corsos y sardos (Dalbera-Stefanaggi 1997; Jones 1997; Loporcaro 1999): las variedades sardas del noroeste (zona del Logudoro) y de la mitad sur (zona del Campidano) se distinguen de la variedad de la zona central-este (zona de Nuoro) por la sonorización y fricativización de las oclusivas sordas intervocálicas (22)a. Las variedades con sonorización de oclusivas sordas evitan la neutralización con la serie sonora por la práctica elisión de estas últimas, frente a la fricativización típica del centro-este $(22) b .{ }^{10}$ La sonorización intervocálica es, en cambio, la norma habitual para las fricativas en todas las variedades (22)c. ${ }^{11}$ Como afirman Giannelli \& Cravens (1997: 39), el hecho de que el proceso siga activo entre palabras (23) sugiere que no ha habido necesariamente una reestructuración del sistema. (Los ejemplos del sardo son de Jones 1997: 320-321.)

(22) Sardo:

$\begin{array}{ll}\text { a. } & {[\operatorname{ludu}]} \\ \text { b. } & {[p e(j)]}\end{array}$

luðu]
Noroccid. y mitad sur

c.

$\begin{array}{lll} & \text { Centro-este } & \\ & {[\text { lutu] }} & \text { 'lodo' } \\ & \text { [peðe] } & \text { 'pie', } \\ \text { [roza], [arroza] } & & \text { 'rosa' }\end{array}$

\footnotetext{
${ }^{10}$ El italiano presenta también casos de sonorización regular de oclusivas en posición intervocálica. En Roma, por ejemplo, se produce una sonorización opcional de las oclusivas intervocálicas $/ \mathrm{t} / \mathrm{y} / \mathrm{k} /$, con solapamiento sustancial con las realizaciones de /d/ y/g/, respectivamente: e.g., pe[g]orino 'queso Pecorino'; también se sonoriza de modo variable la /p/ intervocálica, aunque en este caso la geminación de /b/ evita la neutralización del par /p/ /b/: e.g., Come si chiamavano? [ß]ara[bb]ole? ‘Cómo se llamaban? ¿Parábolas?’ (cf. Hualde \& Nadeu 2011; Hualde 2013, y las referencias que allí se citan). El mantenimiento por vías alternativas del contraste en la serie labial es similar a lo que se observa en la Figura 1: mientras que el alófono correspondiente a la consonante /b/ presenta una estructura de formantes típica de las realizaciones aproximantes, la variante de /p/ sigue siendo oclusiva, preservando así la diferencia.

11 La sonorización de /s/ intervocálica en italiano también es frecuente en muchos dialectos. En la variedad lombarda descrita por Krämer (2009: 207-209), por ejemplo, las sibilantes intervocálicas son sonoras excepto cuando son el elemento inicial de una raíz: e.g., a[z]ola 'ojal', di[z]-uguale 'desigual' vs. a-[s]ociale 'asocial', lo [s]apevo 'lo sabía' (vendesi 'se vende', sin embargo, presenta variación). Sobre esta cuestión, v. también Loporcaro (1995; 1999) y Bertinetto (1999).
} 
(23) Sardo:
a. /sa terra/
b. /sa domo/
c. /su sole/

\begin{tabular}{|c|c|}
\hline $\begin{array}{l}\text { Noroccid.y mitad sur } \\
\text { [sa ðerra }] \\
{[\text { sa omo }] /[\mathrm{su} \text { omu }]} \\
{[\text { su zole }]}\end{array}$ & $\begin{array}{l}\text { Centro-este } \\
\text { [sa terra] } \\
\text { [su ðom॰] } \\
\text { zoli] }\end{array}$ \\
\hline
\end{tabular}

Terminamos el estudio de la sonorización de obstruyentes en el ámbito de la palabra haciendo referencia a la sonorización ocasional y variable que presenta, en el español de la Sierra del Ecuador, /s/ ante pausa en contextos dubitativos: digamo[z]...; los precios de lo[z]... comerciantes (Lipski 1989: 54). La sonorización en este contexto en una lengua sin contraste $/ \mathrm{s} / \sim / \mathrm{z} / \mathrm{se}$ atribuye a la inercia de la vibración de las cuerdas vocales durante la pronunciación de la vocal, que se mantiene durante la producción de la sibilante. Los autores coinciden en subrayar que no se trata, en ningún caso, de un proceso regular fonológico sino de un efecto fonético (similar a la ligera extensión de la vibración de las cuerdas vocales desde la vocal tónica de París hasta el inicio de la /s/ final en la secuencia de la Figura 1).

\section{Sonorización entre palabras}

\subsection{Descripción de los datos}

Entre palabras, las lenguas romances presentan también diferentes procesos de asimilación de la sonoridad. En algunos contextos, existe una solución mayoritaria, como cuando una obstruyente final de palabra precede a una consonante inicial de sílaba en la palabra siguiente: en varias lenguas, la primera consonante se asimila al valor de sonoridad de la segunda, tanto si esta es también una obstruyente (24) como si es una sonante (25)(con simplificaciones adicionales en algunos casos; cf. occitano en (24)b y (25)b); los ejemplos con oclusivas finales son limitados porque en muchas lenguas no existen o son raros (cf., e.g., catalán ciutat -[t]; occitano languedociano marrit - $[\mathrm{t}]$ 'malo' vs. español general ciudad $-[\delta] /-[\theta] /-\varnothing$; portugués, gallego cidade; italiano città; occitano provenzal marrit -Ø).

(24) a. Catalán (estándar)

sap cantar

sap ballar

dues taques

dues vaques $[\mathrm{pk}]$

$[\mathrm{bb}]$

[st]

$[\mathrm{z} \beta]$

'sabe cantar'

'sabe bailar'

'dos manchas'

'dos vacas'

b. Occitano (languedociano; Wheeler 1988)

ne faguèt tres

ne faguèt doas

voldràs tu

avètz jogat [tt]

[dd]

[st]

[dd3] 'hizo tres'

'hizo dos (femenino)'

'querrás tu'

'habéis jugado'

c. Español (estándar)

dos patas

dos vacas

d. Gallego (Freixeiro 2006)

non nos chama

cruz verde

[st

[ð $\beta]$ 'no nos llama'

'cruz verde' 
e. Portugués (europeo; Mateus \& d'Andrade 2000) maus tempos maus dias

$\left[\int \mathrm{t}\right]$

[3d]

[zm]

$[\mathrm{bm}]$

sap mentir

b. Occitano (languedociano; Wheeler 1988)

despartissètz lo vòstre

tap long

[d]zl]

([11])

[zm]

los moños

d. Gallego (Freixeiro 2006)

os nosos

[zn] 'malos tiempos'

'malos días'

'los moños'

'sabe mentir'

'repartid el vuestro'

'tapón largo'

e. Portugués (europeo; Mateus \& d'Andrade 2000) dois livros

[31]

'dos libros'

En italiano, sin embargo, la sibilante /s/ final de palabra se mantiene como sorda ante consonante sonora (26). El rumano sigue el mismo patrón que en el interior de palabra (cf. (11)a) y solo presenta asimilación entre obstruyentes (27). El francés, por su parte, conserva en general el contraste en los casos excepcionales en que las obstruyentes finales - no derivadas de una posible secuencia con vocal pospuesta - se mantienen ante una consonante (28); son excepcionales porque en general las consonantes de coda final se eliden ante otra consonante (six [sis] 'seis', six garçons [sigarsõ] 'seis chicos') mientras que las consonantes de estas palabras se mantienen (bus [bys] 'autobús', bus petit [byspəti] 'autobús pequeño'), aunque limitadas a veces a contextos específicos: avec 'con', sac 'bolso', bec 'pico', donc 'pues (causal)', sens 'sentido', bus, plus '+', tous (pronombre) 'todos'...'

(26) Italiano (Bertinetto 1999)

bus puntuale

lapis blu

(27) Rumano (Cazacu et al. 1967)
a. te rog să vii
am mers de două ori
$[\mathrm{ks}]$
[zd]
b. mă duc la institut
$[\mathrm{kl}]$
m-am dus la teatru
[sl]

$[\mathrm{sp}]$
$[\mathrm{sb}]$

$$
\mathrm{sb]}
$$

'bus puntual'

'lápiz azul'

\author{
'te ruego que vengas' \\ 'he ido dos veces' \\ 'voy al instituto' \\ 'fui al teatro'
}

\footnotetext{
12 La falta de asimilación regular ante obstruyente del francés se puede atribuir a la incorporación de consonantes como la $s$ en tous (28)b al ataque de una sílaba con núcleo vacío, por lo que se mostrarían más reticentes a modificar sus rasgos (v. también n. 13 y $\$ 4.3$ ).
} 
(28)Francés (Peyrollaz \& Bara de Tovar 1954; Sten 1956; Bertinetto 1999)
a. avec toi
bus petit
avec du sable
[kt]
$[\mathrm{sp}]$
$[\mathrm{k} / \mathrm{gd}]$
'contigo'
'autobús pequeño'
'con arena'
b. le sens de l'odorat
ils sont tous venus
[sd]
[sv]
'el sentido del olfato'
c. avec Marie
tous mangent
$[\mathrm{km}]$
$[\mathrm{sm}]$
'han venido todos'
'con María'
'todos comen'

Cuando las obstruyentes finales preceden a una palabra comenzada por vocal, encontramos bastante variación inter- e intralingüística. Para el caso de la sonorización de $s$ intervocálica, contamos con el estudio de Loporcaro $(1995 ; 1999)$ sobre la variación existente en las lenguas romances atendiendo al tipo de límite morfológico en que se halla la sibilante; Loporcaro demuestra que, en general, se sigue un patrón inclusivo según el grado de cohesión del límite: cuanta menor cohesión, menor es la probabilidad de encontrar sonorización. Nuestro estudio lo complementa porque se concentra en la variación existente atendiendo primordialmente al tipo de consonante afectada según el contexto melódico en que aparece y, secundariamente, a su posición en la palabra, sin entrar en la detallada descripción morfológica que presenta Loporcaro. Nuestro propósito es demostrar que la variación detectada en relación con el contexto melódico, lejos de presentar un patrón aleatorio, también se ordena en un esquema inclusivo, de manera que los contextos con sonorización de las variedades más restrictivas son un subconjunto de los contextos de las más permisivas.

Para empezar, en español general, en gallego y en italiano, las obstruyentes sordas finales mantienen el rasgo sordo cuando se resilabean como ataques de la palabra siguiente (29)a-c (en el caso del italiano, algunas consonantes, como la $s$ final de bus, experimentan raddoppiamento en este contexto, cuestión que no tratamos aquí; cf. Krämer 2009: 199); el rumano, por su parte, mantiene el carácter sordo o sonoro de las obstruyentes finales de palabra (29)d. Otras variedades permiten la sonorización de algunas obstruyentes prevocálicas: en portugués y en el español de la Sierra del Ecuador, por ejemplo, las sibilantes sordas finales de palabra se sonorizan regularmente (cf. (30)a y (30)b). El comportamiento del resto de obstruyentes en esta posición es ambiguo, puesto que, como hemos dicho, en gallego, en italiano y en portugués las oclusivas no suelen aparecer en posición final de palabra, mientras que en español tienden a realizarse como aproximantes sonoras, oscureciendo así los posibles procesos de sonorización.

(29)a. Español (estándar) has ido

[asiðo]

b. Gallego (Freixeiro 2006) as aves

[asaßes]

'las aves'

c. Italiano (Bertinetto 1999; Krämer 2009)

herpes incredibile

bus azzurro

[herpesinkredi:bile]
[bussaddzuro]

'herpes increíble'

'autobús azul' 
d.Rumano (Cazacu et al. 1967; Mallinson 1988)

$\begin{array}{ll}\text { sînt în hotel } & \text { [sintinhotel] } \\ \text { cînd a venit? } & \text { [kindavenit] } \\ \text { rus alb } & \text { [rusalb] } \\ \text { autobuz apropae } & \text { [autobuzaproape] }\end{array}$

'están en el parque'
¿cuándo vino?'
'ruso blanco'
'autobús cerca'

(30)a. Español de la Sierra del Ecuador (Lipski 1989) has ido [aziðo]

b. Portugués (europeo; Mateus \& d'Andrade 2000) maus amigos [mawzemiguf] 'malos amigos'

En francés, los numerales que terminan con fricativa final sibilante (six 'seis', dix 'diez') o labiodental (neuf 'nueve') asimilan la sonoridad en posición prevocálica en construcciones que podrían ser consideradas, por ello, de liaison (31)a, como sugiere Sten (1956: 60); para neuf, solo en los sintagmasneuf heures y neuf ans. ${ }^{13}$ En cambio, las palabras con oclusivas o fricativas finales excepcionales ilustradas anteriormente en (28) (esto es, las que se conservan en todos los contextos) mantienen esta consonante como sorda (31)b-c.

(31) Francés (Peyrollaz \& Bara de Tovar 1954; Sten 1956; Bertinetto 1999)

\begin{tabular}{|c|c|c|}
\hline six heures & [sizœь] & 'las seis' \\
\hline six amis & [sizami] & 'seis amigos' \\
\hline neuf heures & [nœvœь] & 'las nueve' \\
\hline six garcons & [sigarsõ] & 'seis chicos' \\
\hline neuf amis & [nœfami] & 'nueve amigos' \\
\hline neuf garcons & [пøgавsо̃] & assõ] 'nueve chicos' \\
\hline b. avec elle & [avekel] & 'con ella' \\
\hline sac orange & [sakoьãn3] & 'bolso naranja' \\
\hline c. tous ensemble & [tusãsãbl] & 'todos juntos' \\
\hline bus orange & [bуsэьа̃з] & 'autobús naranja' \\
\hline tous mangent & [tusmãz] & 'todos comen' \\
\hline bus grand & [bysgка̃] & 'autobús grande' \\
\hline
\end{tabular}

El patrón de variación del catalán en (32) presenta una gradación claramente inclusiva. El valenciano central es la variedad menos proclive a la asimilación y mantiene sin sonorizar todas las obstruyentes finales de palabra (32)a, como el gallego, el italiano y el español general (29). El valenciano general (32)b y el catalán hablado en la ciudad sarda de Alghero (i.e. alguerés) presentan sonorización, pero únicamente de la conso-

\footnotetext{
13 No profundizamos, por cuestión de espacio y por la complejidad de los datos, ni en las consonantes de liaison (е.g., vous [vu] 'vosotros' vs. vous allez [vuzale] 'vosotros vais', grand [gкã] 'gran (masculino)' vs. grand ami [ука̃tami] 'gran amigo', y en algún numeral), ni en las consonantes finales que se consideran ataque de una sílaba con 'e muda' (núcleo [ə] en los dialectos meridionales) (grande [gьã.də] 'gran (femenino)', grande amie [gьã.da.mi] 'gran amiga'), ni en los grupos secundarios - admitidamente muy variables derivados de la elisión de $e$ en la concatenación de palabras (la queue de ce renard 'la cola de este zorro'

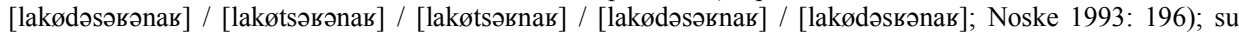
interpretación depende, en todo caso, de la estructura prosódica que se presume en cada construcción (v., entre otros, Noske 1993: §6.2 para una revisión de los distintos tipos de alternancias [ə] Ø que presenta el francés). Tan solo presentamos, para ilustrar nuestra línea de análisis, algunos casos de elisión regular de $e$ dentro de la palabra y en el grupo clítico en $\$ 4.3$.
} 
nante sibilante [s], replicando así el patrón del portugués y del español de la Sierra del Ecuador (30). En catalán central, se asimilan regularmente todas las sibilantes, incluidas las africadas, mientras que la fricativa labiodental $f$ se sonoriza de manera variable (32)c. ${ }^{14}$ Finalmente, en la variedad alicantina, la menos restrictiva, todas las consonantes finales, incluso las oclusivas, se sonorizan (32)d.

(32) Gradación de la asimilación de consonantes finales prevocálicas en catalán
a. Valenciano central
gos ideal
vaig a casa
[gosiðeal]
[vatfakasa]
xef únic
[t]efunik]
bec aigua
[bekajywa]
'perro ideal'
'voy a mi casa'
'chef único'
'bebo agua'
b. Valenciano general (y alguerés)

$\begin{array}{ll}\text { gos ideal } & \text { [goziðeal] } \\ \text { vaig a casa } & \text { [vatfakaza] } \\ \text { xef únic } & \text { [t } \text { fefunik] } \\ \text { bec aigua } & \text { [bekajywa] }\end{array}$
'perro ideal'
'voy a mi casa'
'chef único'
'bebo agua'
c. Catalán central (y, por extensión, catalán estándar)

$\begin{array}{ll}\text { gos ideal } & \text { [goziðeal] } \\ \text { vaig a casa } & {[\text { baḑəkazə] }} \\ \text { xef únic } & {\left[\int \varepsilon f / \text { vunik] }\right.} \\ \text { bec aigua } & {[\text { bekajðwə] }}\end{array}$
'perro ideal'
'voy a mi casa'
'chef único'
'bebo agua'

\section{d. Alicantino \\ gos ideal \\ vaig a casa \\ xef únic \\ bec aigua}

$$
\begin{aligned}
& \text { [goziðeal] } \\
& \text { [vadzakaza] } \\
& \text { [t]evunik] } \\
& \text { [begajywa] }
\end{aligned}
$$
'perro ideal'
'voy a mi casa'
'chef único'
'bebo agua'

El occitano (languedociano), finalmente, presenta un patrón como el del catalán central (cf. (32)c): las oclusivas finales prevocálicas, en los dialectos que no las eliden, se mantienen sordas (33)a; las fricativas y las africadas prevocálicas entre palabras se sonorizan (33)b. ${ }^{15}$

(33) Occitano (languedociano; Wheeler 1988; Alibèrt 2000; Carrera 2011)
a. diguèt a son paire
[digetasumpajre]
b. siás un marrit
[sjozymmarit]
puèg agut
[pedzagyt]
'dijo a su padre'
'eres una mala persona'
'pico agudo'

En los siguientes apartados, analizamos la naturaleza de la restricción que favorece la asimilación entre palabras (\$4.2). A continuación, discutimos la manera de formalizar, en un modelo paralelo de TO, la relación entre la asimilación prevocálica entre palabras y los principios de fidelidad (§4.3). Finalmente, estudiamos cómo se puede dar

\footnotetext{
${ }^{14}$ Sobre el comportamiento ambivalente de $f$, como oclusiva o como fricativa, en distintos fenómenos fonológicos del catalán (y otras lenguas), v. Lloret\& Pons \& Jiménez (2011).

15 Aitor Carrera (c. p.) nos indica que las pocas palabras que terminan en $-f$ se tendrían que sonorizar ante vocal, pero que en el habla habitual añaden una vocal (filosòfe, en vez de filosòf 'filósofo').
} 
cuenta de los efectos graduales observados en la asimilación de las lenguas romances $(\S 4.4)$.

\subsection{El imperativo asimilatorio}

Para comprender la variación observada en los datos anteriores, la primera cuestión que tenemos que aclarar es la naturaleza del principio que provoca la asimilación entre palabras. Si las lenguas estudiadas se limitaran a permitir en ese entorno la asimilación en grupos consonánticos, serían suficientes los principios AGREE $[ \pm \text { voice }]_{\text {obob }} \mathrm{y}$ AGREE $[ \pm \text { voice }]_{\mathrm{ObN}}$, que hemos definido en $\S 3.2$; las restricciones de fidelidad referidas a los ataques silábicos, tanto dentro de la palabra como entre palabras, asegurarían que la concordancia se realizara seleccionando los rasgos asociados al ataque. El cuadro se complica entre palabras por la sonorización de algunas obstruyentes prevocálicas en lenguas como el catalán (igual que ocurre, en secuencias similares, en portugués, en occitano y, con diferentes limitaciones, en el español de la Sierra del Ecuador y en francés).

Nuestra propuesta para formalizar la sonorización entre palabras se basa en la observación de que, en general, los segmentos que causan la asimilación suelen tener mayor relevancia perceptiva que los segmentos asimilados. En los grupos de obstruyentes, en la palabra o entre palabras, la diferencia se debería al silabeo de las consonantes: la primera - la asimilada -, en la posición débil de coda, y la segunda, en la posición prominente del ataque silábico. En el caso de las obstruyentes prevocálicas en posición final de palabra (y, como efecto añadido, en los grupos de obstruyentes repartidos entre palabras), el contraste posicional se inferiría de la prominencia relativa de la posición inicial de palabra respecto de los elementos previos, recogida por la restricción de (34).

(34) AGREE[ $[ \pm \text { voice }]_{\text {In }}$ : Una obstruyente y un segmento inicial de palabra deben concordar en el rasgo $[ \pm$ sonoro $] .{ }^{16}$

La formulación de (34) implica que, en nuestro tratamiento, el rasgo [+sonoro] se propaga desde la vocal inicial de palabra. En efecto, aunque seguramente el proceso de sonorización de consonantes finales prevocálicas se pueda haber originado en posición intervocálica, un contexto en que las sonoras podrían considerarse un elemento poco marcado (cf. §3.3), los ejemplos de (35) muestran que, al menos para algunas lenguas, la asimilación de las sibilantes se ha generalizado a todos los contextos prevocálicos, incluyendo aquellos en que estas aparecen tras una sonante o una obstruyente. La sonorización en estos casos, en que no existen condicionantes fonéticos tan evidentes, indica que nos encontramos ante un fenómeno fonológico, causado por la vocal inicial de la palabra siguiente. De hecho, si en catalán central, por ejemplo, el rasgo se extendiera desde la vocal previa en sacs ideals [...agzi...] (/sakz idealz/) 'sacos ideales', o desde las dos vocales a la vez, esperaríamos también asimilación en sac ideal (/sak ideal/) [...aki...] (*[...agi...]). Igualmente, en el español de la Sierra del Ecuador la elisión de vocales en la sílaba final de palabras como ustedes conduce a la sonorización de la sibilante entre palabras en un contexto no estrictamente intervocálico. ${ }^{17}$

\footnotetext{
16 AGREE[ \pm voice $]_{\text {In }}$ se puede subdividir también en función de la distancia articulatoria entre los dos segmentos implicados, una cuestión sobre la que volveremos en $\$ 4.4$.

17 Discrepamos, así, de trabajos recientes que a la vista del posible origen analógico de las sonorizaciones intervocálicas concluyen que el análisis fonológico sincrónico tiene que acometerse inexorablemente por
} 
(35) a. Catalán central

$\begin{array}{ll}\begin{array}{l}\text { gos ideal } \\ (/ \mathrm{gos} /)\end{array} & \text { [goziðeal] } \\ \text { fals ideal } & \\ (/ \mathrm{fals} /) & \text { [falziðeal] } \\ \begin{array}{l}\text { sacs ideals } \\ (/ \mathrm{sakz} /)\end{array} & \text { [sagziðeals] } \\ \begin{array}{l}\mathrm{ff} . \mathrm{sac} \text { ideal } \\ (/ \mathrm{sak} /)\end{array} & \text { [sakiðeal] }\end{array}$

b. Español de la Sierra del Ecuador (Lipski 1990)

usted(e)[z] allá

oyent(e)[z] americanos

\subsection{Fidelidad más allá de la palabra}

La diferencia entre secuencias léxicas (e.g., catalán oci [si] 'ocio'; español sido [si]) y postléxicas (e.g., catalán gos ideal [zi]; español de la Sierra del Ecuador has ido [zi]) supone un problema de opacidad, típico de versiones paralelas de modelos fonológicos como la TO clásica: que las consonantes asimiladas ocupen la posición de coda en las palabras aisladas (catalán gos; español has) es esencial para que se puedan sonorizar, pero esta información contextual, que justificaría la (sobre)aplicación del proceso, desaparece en el resultado superficial, ya que los segmentos finales se resilabean como ataques. Por consiguiente, la mayoría de trabajos que abordan la sonorización entre palabras analizan el fenómeno por medio de versiones cíclicas o por estratos de la fonología, tanto en fonología autosegmental (Mascaró 1987; Lipski 1989) como en TO (e.g., Jiménez 1999; Bermúdez-Otero 2001; 2006; 2011; Bradley 2005; Bradley \& Delforge 2006; Beckman \& Ringen 2007; 2008), y lo hacen apelando a un estadio intermedio, problemático, en que la consonante final aparece en la coda y pierde (o no tiene) la especificación para el rasgo $\left[{ }^{ \pm}\right.$sonoro]. Por nuestra parte, siguiendo el trabajo iniciado en Jiménez \& Lloret (2008) para el catalán, tomamos el reto de formalizar esta memoria estructural de las consonantes finales dentro de la versión clásica paralela de TO. ${ }^{18}$

Como punto de partida para el análisis, supondremos que la asimilación en gos ideal está causada por la restricción específica AGREE[ \pm voice $]_{\text {In }}$ (cf. §4.2). Si este principio se situara por debajo del principioIDENT ${ }_{\mathrm{ONS}}$, seguiría sin haber asimilación en el límite entre palabras: como mostramos en (36), IDENT $_{\mathrm{ONS}}$, desde su posición dominante en la jerarquía, evitaría la asimilación.

(36)/gos ideal/ (catalán)

\begin{tabular}{|c|c|c|c|}
\hline$/ \ldots \mathrm{si} \ldots /$ & IDENT $_{\text {ONS }}$ & AGREE $[ \pm \text { voice }]_{\text {In }}$ & IDENT \\
\hline a. ...si... & & $*$ & \\
\hline b. ...zi... & $* !$ & & $*$ \\
\hline
\end{tabular}

Igualar el tratamiento de las secuencias léxicas y postléxicas implica, sin embargo, un problema conceptual de fondo, puesto que así se otorga la misma protección y, por lo

niveles (Strycharczuk et al. 2013) y/o derivarse de la extensión del rasgo [+sonoro] de la vocal precedente (Strycharczuk \& Simon 2013).

18 Wheeler (2005) y Colina (2009) presentan también análisis en paralelo de la sonorización prevocálica en catalán y en el español de la Sierra del Ecuador, respectivamente 
tanto, la misma relevancia, a los segmentos que se silabean dentro de la palabra en posiciones distintivas y a los segmentos que aparecen al final de la palabra en posiciones no distintivas. Es decir, en un análisis como el de (36)elementos que no permiten el contraste de sonoridad a final de palabra - las consonantes finales - son tratados como distintivos más allá de la palabra.

Para garantizar que únicamente los elementos asociados a los ataques tengan siempre un papel preeminente en todos los niveles, se ha sugerido que el mantenimiento de los rasgos en el nivel postléxico esté gobernado por restricciones del tipo OUTPUTOUTPUT(OO)-FAITHFULNESS, que exigen la igualdad superficial entre todas las ocurrencias de un mismo ítem léxico (fonología transderivacional; Benua 1997). Como en la palabra, las restricciones OO también se desglosan, en función de la prominencia posicional de los elementos, en un principio que exige la igualdad entre los segmentos asociados al ataque en la base, OO-IDENT ${ }_{\mathrm{ONS}}$, $\mathrm{y}$ un principio general que exige la correspondencia de todos los rasgos, OO-IDENT (ordenación universal:OO-IDENT ${ }_{\text {ONS }}>>$ OOIDENT, ambos probablemente por encima del par IDENT ${ }_{\text {ONS }}>>$ IDENT).

El comportamiento de una lengua como el catalán, que permite la sonorización de todas las obstruyentes de las codas a una consonante siguiente, en la palabra o entre palabras, y también la sonorización de alguna consonantes finales de palabra en posición prevocálica, se infiere de la jerarquía compuesta OO-IDENT ${ }_{\text {ONS }}$ $>>$ AGREE[ $[ \pm \text { voice }]_{\text {ObC }}$, AGREE[ \pm voice $]_{\text {In }}>>$ OO-IDENT $\left[>>\right.$ IDENT $_{\text {ONS }}>>$ IDENT] (la restricción AGREE[ $[ \pm \text { voice }]_{\mathrm{ObC}}$ agrupa la tendencia a asimilar obstruyentes ante obstruyentes, AGREE $[ \pm \text { voice }]_{\mathrm{ObOb}}, \mathrm{y}$ ante sonantes, AGREE $\left.[ \pm \text { voice }]_{\mathrm{ObN}}\right)$. Con esta jerarquía, en (37) es elegido el candidato con asimilación prevocálica de la sibilante final, puesto que este segmento ocupa una posición no distintiva en la base (esto es, en la realización de la palabra aislada: [gos]) y sus rasgos no son protegidos de la influencia de AGREE $[ \pm \text { voice }]_{\text {In }}$ por el principio OO-IDENT ${ }_{\text {ONS }} \cdot{ }^{19}$

(37)/gos ideal/ (catalán); bases: [gos], [iðeal]

\begin{tabular}{|c|c|c|c|}
\hline /...si.../ & OO-IDENT $_{\text {ONS }}$ &  & OO-IDENT \\
\hline a....si... & & $* !$ & \\
\hline b. ...zi... & & 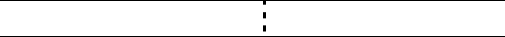 & * \\
\hline
\end{tabular}

La asimilación al elemento inicial de la palabra afecta también a grupos finales de obstruyente y sibilante, como muestra el cuadro de 0: la falta de asimilación en 0a, que se corresponde exactamente con el resultado para sacs aisladamente (con ensordecimiento final: [saks]), es descartada a favor de la extensión del rasgo sonoro del elemento inicial de la palabra $0 \mathrm{~b}$. La obstruyente velar se asimila también por el efecto del principio más general AGREE $[ \pm \text { voice }]_{\mathrm{ObC}}$.

19 Con la ordenación de (37) también se sonorizarían - incorrectamente en algunos casos - las oclusivas finales en secuencias como sac ideal [ki] o bec aigua [kə]; más adelante, en $\$ 4.4$ abordamos la manera de atenuar el papel de AGREE[ \pm voice $]_{\text {In }}$ en la jerarquía con el fin de evitar estos resultados. 
(38)/sakz idealz/ (catalán); bases: [saks], [iðeals]

\begin{tabular}{|c|c|c|c|c|}
\hline /...akz i.../ & OO-IDENT $_{\mathrm{ONS}}$ & AGREE $[ \pm \text { voice }]_{\mathrm{ObC}}$ & AGREE $[ \pm \text { voice }]_{\text {In }}$ & OO-IDENT \\
\hline a. ...aksi... & & & $* !$ & \\
\hline b. ....agzi... & & & & ** \\
\hline c. ...akzi... & & $* !$ & & * \\
\hline
\end{tabular}

El tratamiento propuesto implica, pues, que las consonantes que ocupan una posición distintiva en un nivel conservan memoria de este carácter en niveles superiores, y a la inversa, si un segmento se silabea en una posición en que sus rasgos pierden el carácter distintivo, siguen sin tenerlo en otros niveles. Al margen de explicar el contraste entre la falta de asimilación en ataques léxicos, como en oci [si], y la asimilación en ataques postléxicos, como en gos ideal [zi], esta propiedad del sistema permite justificar el mantenimiento de las especificaciones subyacentes en los ejemplos de (39), del catalán y del portugués, respectivamente.

(39) a. Catalán estándar fer-se altes

[fersaltəs]

'hacerse altas'

b. Portugués (Mateus \& d'Andrade 2000: 145-146)

disse à Nita

[disanite]

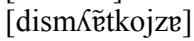

'dijo a Nita' disse semelhante coisa

'dijo semejante cosa'

En los ejemplos anteriores, la elisión de una vocal provoca que una sibilante sorda situada en el ataque en el nivel léxico pase a ocupar una posición - final de palabra prevocálica, en catalán y en el primer ejemplo del portugués; preconsonántica, en el segundo ejemplo del portugués - en que este tipo de consonantes suelen sonorizarse. La necesidad de respetar las especificaciones léxicas de los ataques, promovida por el principio OO-IDENT ${ }_{\mathrm{ONS}}$, es de nuevo la responsable del mantenimiento de los rasgos en (40), a pesar de que la consonante resilabeada se encuentre superficialmente en una posición estructural prácticamente idéntica a la que hemos descrito en (37).

(40)/dise a nita/ (portugués); bases: [disi], [a], [nite]

\begin{tabular}{|c|c|c:c|c|}
\hline$/$ dise a nita/ & OO-IDENT $_{\mathrm{ONS}}$ & AGREE$\left[ \pm\right.$ Voice $_{\mathrm{ObC}}$ & AGREE[ \pm voice $]_{\mathrm{In}}$ & $\begin{array}{c}\text { OO- } \\
\text { IDENT }\end{array}$ \\
\hline a. disanite & & & $*$ & \\
\hline b. dizanite & $* !$ & & & $*$ \\
\hline
\end{tabular}

El análisis que acabamos de presentar deriva los rasgos extendidos y preservados de las asimetrías perceptivas entre los elementos que interactúan: como norma general, se extienden - y se conservan - los rasgos asociados a posiciones relativamente prominentes. Este axioma ayuda a comprender mejor un ejemplo de dirección variable en la asimilación: en francés, como muestran los ejemplos de 0 , la elisión de algunas vocales provoca la aparición de grupos consonánticos secundarios. 
(41)Francés (Walker 1984)

a. Dentro de la palabra cheval semaine

[ff]

[sm]

b. Entre el proclítico y su huésped

je sais

je fais

je pars $\left[\int \mathrm{s}\right] \rightarrow\left[\int\right]$

[Sf]

[Sp] 'caballo'

'semana'

'yo sé'

'yo hago'

'yo me voy'

Los grupos de consonantes creados por la elisión concuerdan en la especificación

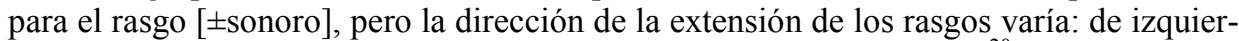
da a derecha dentro de la palabra y de derecha a izquierda en proclisis. ${ }^{20}$ Aunque puedan parecer contradictorios, ambos patrones comparten una misma propiedad, ya observada en el análisis de (37): se extiende siempre la especificación del segmento inicial de palabra, más prominente que las consonantes del interior de la palabra en 0a. Por otra parte, aunque las consonantes asimiladas de 0b ocupan inicialmente una posición de ataque inicial de palabra, pertenecen a un elemento clítico, secundario también respecto del verbo al que se adjunta, cuya consonante inicial gobierna la asimilación.

El mecanismo de análisis propuesto, con pugnas entre fidelidades input-output y output-output y cambios por cuestiones asimilatorias de marcación, también sirve para explicar el carácter sordo de las sibilantes finales excepcionales del francés (bus, tous, sens, etc.; cf. (31)), que se mantienen en posición prevocálica y preconsonántica entre palabras: si derivamos el carácter excepcional de estas consonantes de la presunción de que ocupan estructuralmente la posición de ataque de una sílaba con núcleo vacío, el rasgo [ - sonoro] de la consonante final de la base (i.e. de la forma fonética de la palabra aislada) puede quedar preservado por fidelidad al ataque, como en tou[s] ensemble (fiel a la base: [tous]); le sen[s] de l'odorat (fiel a la base: [sãs]). ${ }^{21}$ Crucialmente, para los casos de asimilación dentro de la palabra y en el grupo clítico vistos en 0 , la inexistencia de una base de referencia en el primer caso (porque se trata de la palabra aislada) y la preponderancia de la base verbal en el segundo permiten los efectos asimilatorios anotados.

\subsection{Efectos graduales en la asimilación}

La gradación de los contextos asimilatorios ilustrada en $\S 4$ guarda semejanza con los patrones que hemos descrito en §3.2: en ambos casos observamos que, cuanto más parecidos son dos segmentos desde el punto de vista articulatorio, más posibilidades exis-

\footnotetext{
20 Walker (1984: 37) interpreta estas asimilaciones como «a third type of assimilation, conditioned by the difference between strong (= voiceless) and weak (= voiced) obstruents. In this case, the weak segment assimilates to the strong, regardless of their relative position. Thus, in je sais, weak $/ \mathrm{z} / \mathrm{precedes}$ strong $/ \mathrm{s} /$ while in cheval, weak /v/ follows strong / $/$ /. The weak consonant becomes voiceless in both cases [...]». Otros ejemplos de asimilación, en que una consonante sorda puede asimilarse a una consonante sonora inicial de palabra, como en avec du sable [k/gd] 'con arena' (cf. (28)), sugieren que el fenómeno puede ser interpretado como asimilación al segmento inicial de palabra.

21 En la misma línea, para dar cuenta del comportamiento excepcional de las oclusivas del portugués en ejemplos sin asimilación como adquirir o absurdo (cf. (9)c), Mateus \& d'Andrade (2000: 45) sugieren que «there is an empty nucleus between the two consonants that prevents the consonant triggering the assimilation rule». Así, solo las sibilantes preconsonánticas de palabras como esbirro ocuparían la coda y se asimilarían a la consonante siguiente.
} 
ten de que interactúen. Para dar cuenta de esta tendencia, en $§ 3.2$ hemos desglosado el principio AGREE[ \pm voice] en función de la proximidad articulatoria entre los elementos que se asimilan; lo mismo podemos hacer en las secuencias postléxicas con la restricción AGREE $[ \pm \text { voice }]_{\text {In }}$. En los contactos consonánticos entre palabras, los efectos de AGREE[ $[ \pm \text { voice }]_{\mathrm{ObOb}} \mathrm{y}$ AGREE$[ \pm \text { voice }]_{\mathrm{ObN}}$, por un lado, y de AGREE $[ \pm \text { voice }]_{\text {In }}$, por el otro, se solapan, por lo que no desglosaremos AGREE[ \pm voice $]_{\text {In }}$ para estos contactos, sino que asumiremos que los dos primeros principios - que resumimos de nuevo, cuando conviene, como AGREE $[ \pm \text { voice }]_{\mathrm{ObC}}$ - cubren ya ese papel. En las secuencias prevocálicas, en cambio, sí que es necesario desglosar AGREE[ \pm voice $]_{\text {In }}$ para algunas lenguas.

En la Tabla 1 resumimos las posibilidades de asimilación en las lenguas romances, desde las variedades más restrictivas hasta las variedades con menos limitaciones (en negrita marcamos las restricciones que favorecen la concordancia). Comenzamos con el italiano, una lengua con asimilación de grupos de obstruyentes en la palabra, pero no entre palabras, y sin asimilación de obstruyentes prevocálicas entre palabras. En un caso así, se ha de suponer que el principio OO-IDENT domina a las restricciones a favor de la asimilación AGREE $[ \pm \text { voice }]_{\text {In }}$ y AGREE $[ \pm \text { voice }]_{\mathrm{ObC}}$ (que a su vez dominarían a $\operatorname{IDENT}_{\mathrm{ONS}}$ y a IDENT). De este modo, las obstruyentes preconsonánticas se asimilan en la palabra pero las especificaciones de las consonantes finales de palabra se mantienen siempre en el nivel postléxico.

Entre palabras, el rumano es un poco menos restrictivo que el italiano, puesto que sitúa AGREE $[ \pm \text { voice }]_{\text {ObOb }}$ (pero no AGREE[ $[ \pm \text { voice }]_{\mathrm{ObN}}$ ) por encima de OO-IDENT, de manera que permite la asimilación en los grupos de obstruyentes en todos los contextos; dentro de la palabra, la ubicación de AGREE[ \pm voice $]_{\text {ObN }}$ por debajo incluso de la restricción IDENT evita la asimilación de obstruyentes ante consonantes sonantes. Si, alternativamente, se modifica la jerarquía del italiano de modo que OO-IDENT sea dominada por la restricción general AGREE[ \pm voice $]_{\mathrm{ObC}}$, se obtienen variedades como el español general, el gallego y el valenciano central, que asimilan las obstruyentes preconsonánticas regularmente, pero no presentan sonorización de consonantes finales prevocálicas.

Para las siguientes variedades es necesario desglosar AGREE[ \pm voice $]_{\text {In }}$ en función de la proximidad articulatoria entre la consonante final y la vocal.Siguiendo esta idea, la exigencia de concordancia entre una fricativa y una vocal es más intensa que no cuando la consonante es oclusiva, tal como recoge la jerarquía desglosada AGREE[ \pm voice $]_{\text {In- }}$ $\mathrm{sV}>$ AGREE $[ \pm \text { voice }]_{\text {In-TV. }}$. Cuando el subprincipio AGREE $[ \pm \text { voice }]_{\text {In-SV }}$ (pero no AGREE[ $[ \pm \text { voice }]_{\text {In-TV }}$ ) se sitúa por encima de OO-IDENT, encontramos variedades que solo permiten la sonorización de las fricativas sibilantes en posición prevocálica, como el español de la Sierra del Ecuador, el portugués, el valenciano general y el alguerés; en las dos últimas variedades las africadas finales y las fricativas labiodentales son tratadas como oclusivas y no se asimilan. Si, por el contrario, AGREE[ \pm voice $]_{\text {In }}$ en general - esto es, todas sus variantes, incluidaAGREE $[ \pm \text { voice }]_{\mathrm{In}-\mathrm{TV}}$ - domina a la restricción OO-IDENT, se sonorizan incluso las oclusivas prevocálicas, como ocurre en alicantino. El catalán central y el occitano languedociano ocupan un punto intermedio entre las dos opciones anteriores, porque permiten asimilar las sibilantes fricativas y las africadas (A). Para formalizar esta opción se puede suponer que en la interpretación de las africadas de estas variedades predomina la parte fricativa del grupo, y por tanto se asimilan (la labiodental tiene un comportamiento ambivalente, y se asimila variablemente). Por simplicidad expositiva, en la Tabla 1 recogemos esta coyuntura con la restricción AGREE[ $[ \pm \text { voice }]_{\text {In-SV/AV. }}$ 
La jerarquía del francés no difiere mucho de la que hemos descrito para el catalán central. La diferencia básica es que las obstruyentes solo se sonorizan ante obstruyentes, por lo que habría que suponer que en esta lengua, como en rumano, únicamente AGREE[ \pm voice $]_{\mathrm{ObOb}}$ domina a OO-IDENT (y no, como en catalán, la versión general referida a las consonantes, AGREE[ $\left.[ \pm \text { voice }]_{\mathrm{ObC}}\right)$. También se pueden sonorizar las fricativas prevocálicas entre palabras, por la acción de AGREE[ \pm voice $]_{\text {In-SV. }}$ En ambos casos, la presencia de OO-IDENT ${ }_{\mathrm{ONS}}$ en la parte superior de la jerarquía garantiza que no se sonoricen las consonantes finales de palabra cuando se interpretan como elementos incorporados al ataque silábico en la palabra aislada.

\begin{tabular}{|c|c|c|}
\hline Variedad & Ordenación de restricciones & Efecto entre palabras \\
\hline - italiano & 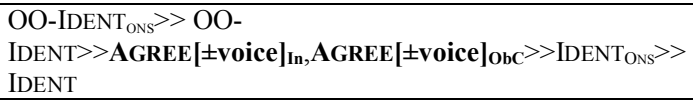 & $\begin{array}{l}\text { - Obstruyentes finales de palabra } \\
\text { no se asimilan }\end{array}$ \\
\hline - rumano & $\begin{array}{l}\text { OO-IDENT }_{\text {ONS }}>>\text { AGREE }[ \pm \text { voice }]_{\mathrm{ObOb}}>>\text { OO- } \\
\text { IDENT }>>\text { AGREE }[ \pm \text { voice }]_{\text {In }}>>\text { IDENT }_{\text {ONS }}>> \\
\text { IDENT }>>\text { AGREE }[ \pm \text { voice }]_{\text {Obn }}\end{array}$ & $\begin{array}{l}\text { - Obstruyentes finales de palabra } \\
\text { se asimilan ante consonante obs- } \\
\text { truyente }\end{array}$ \\
\hline $\begin{array}{l}\text { - esp. gen. } \\
\text { - gallego } \\
\text { - val. central } \\
\end{array}$ & 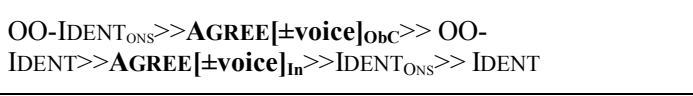 & $\begin{array}{l}\text { - Obstruyentes finales de palabra } \\
\text { se asimilan ante consonante }\end{array}$ \\
\hline - francés &  & $\begin{array}{l}\text { - Obstruyentes finales de palabra } \\
\text { se asimilan ante obstruyente (ex- } \\
\text { cepto las que son ataques en la } \\
\text { palabra aislada) } \\
\text { - Fricativas finales de palabra se } \\
\text { sonorizan ante vocal (excepto las } \\
\text { que son ataques en la palabra ais- } \\
\text { lada) ( } f \text { según la construcción) }\end{array}$ \\
\hline $\begin{array}{l}- \text { val. gen. y } \\
\text { alg. } \\
\text { - esp. (Ecua- } \\
\text { dor) } \\
- \text { portugués }\end{array}$ & $\begin{array}{l}\text { OO-IDENT }{ }_{\mathrm{ONS}}>>\text { AGREE }[ \pm \text { voice }]_{\mathrm{ObC}}, \text { AGREE }[ \pm \text { voice }]_{\text {In-Sv }}>> \\
\text { OO-IDENT }>>\text { AGREE }[ \pm \text { voice }]_{\text {In-TV }}>>\text { IDENT }_{\mathrm{ONS}}>>\text { IDENT }\end{array}$ & $\begin{array}{l}\text { - Obstruyentes finales de palabra } \\
\text { se asimilan ante consonante } \\
\text { - Sibilantes fricativas finales de } \\
\text { palabra se sonorizan ante vocal }\end{array}$ \\
\hline $\begin{array}{l}\text { - cat. central } \\
\text { - occ. lang. }\end{array}$ & $\begin{array}{l}\text { OO-IDENT }{ }_{\text {ONS }}>>\text { AGREE }[ \pm \text { voice }]_{\text {obC }}, \text { AGREE }[ \pm \text { voice }]_{\text {In- }} \\
\text { sv/AV }>>\text { OO-IDENT }>>\text { AGREE }[ \pm \text { Voice }]_{\text {In-TV }}>>\text { IDENT }_{\text {ONS }}>> \\
\text { IDENT }\end{array}$ & $\begin{array}{l}\text { - Obstruyentes finales de palabra } \\
\text { se asimilan ante consonante } \\
\text { - Sibilantes (fricativas y africadas) } \\
\text { finales de palabra se sonorizan } \\
\text { ante vocal (y } f \text { variablemente) }\end{array}$ \\
\hline - alicantino & $\begin{array}{l}\text { OO-IDENT }{ }_{\text {ONS }}>>\text { AGREE }[ \pm \text { voice }]_{\text {ObC }}, \text { AGREE }[ \pm \text { voice }]_{\text {In }}>> \\
\text { OO-IDENT }>>\text { IDENT }_{\text {ONS }}>>\text { IDENT }\end{array}$ & $\begin{array}{l}\text { - Obstruyentes finales de palabra } \\
\text { se asimilan ante consonante } \\
\text { - Obstruyentes finales de palabra } \\
\text { se sonorizan ante vocal }\end{array}$ \\
\hline
\end{tabular}

Tabla 1. Efectos graduales de la asimilación entre palabras (las restricciones en negrita favorecen la concordancia; las restricciones en fina, la fidelidad al input).

\section{Conclusión}

Los efectos inclusivos detectados en la sonorización de obstruyentes de las lenguas romances refuerzan, por un lado, las propuestas que tienen en cuenta el grado de similitud articulatoria entre los segmentos asimilados y, por otro, la interpretación de este fenómeno como asimilatorio en los contextos prevocálicos en posición final de palabra, para los que aun existe cierta polémica sobre la identificación de las causas que provocan el cambio, tan común por otra parte entre las lenguas del mundo. A la diferencia 
articulatoria entre los segmentos se puede atribuir, en primer lugar, la posibilidad de que los grupos de obstruyente y sonante se presenten sin concordancia en lenguas como el rumano o el francés, mientras que los grupos de obstruyentes tienden a igualarse mayoritariamente. Asimismo, la solución basada en la gradación ofrece una respuesta satisfactoria a la peculiaridad de que, aunque la tipología universal favorezca las distinciones de sonoridad en la serie oclusiva antes que en la fricativa (Maddieson \& Ladefoged 1996), en las lenguas romances actuales se sonoricen las fricativas intervocálicas entre palabras antes que las oclusivas, tanto si las lenguas disponen de contraste léxico en la serie (e.g., catalán, francés, occitano, portugués) como si no (e.g., español de la Sierra del Ecuador). Queda fundamentado además el comportamiento fonológico ambiguo de las africadas y de la fricativa labiodental, porque por sus características articulatorias se agrupan ora con las fricativas ora con las oclusivas en cuanto a los fenómenos de sonorización (y a otros fenómenos no estudiados aquí). Finalmente, el análisis que presentamos justifica el carácter fonológico de la sonorización prevocálica, sin negar su posible origen fonético-contextual ni su presencia inestable en algunas variedades y en contextos particulares. En definitiva, sitúa el fenómeno de la sonorización de las obstruyentes prevocálicas en un peldaño más de la escala que contiene los distintos contextos en que la asimilación de sonoridad actúa. Una vez más, los datos revelan la variación ordenada que las lenguas presentan, en su intento de mantener un equilibrio más o menos estable entre la tendencia a la simplificación articulatoria y la necesidad de preservar los contrastes para mantener las distinciones.

\section{Bibliografía}

ALIBÈRT, Loís (2000). Gramatica occitana (segon los parlars lengadocians). Facsímil de la $2^{\text {a }}$ ed. de 1976. Barcelona / Tolosa, Institut d'Estudis Occitans.

AlmeIDA SuÁrEZ, Manuel (1982). «En torno a las oclusivas tensas gran canarias». Revista de Filología de la Universidad de la Laguna1: 77-88

ARCHANGELI, Diana (1988). «Aspects of underspecification theory». Phonology 5: 183207.

BAERTSCH, Karen (2002). An Optimality Theoretic Approach to Syllable Structure: The Split Margin Hierarchy. Tesis doctoral. Indiana University, Bloomington.

BAERTSCH, Karen \& DAVIS, Stuart (2003). «The split margin approach to syllable structure». ZAS Papers in Linguistics 32: 1-14

Beckman, Jill N. (1998). Positional faithfulness. Tesis doctoral. University of Massachusetts, Amherst. Publicada en 1999: Positional faithfulness: an optimality theoretic treatment of phonological asymmetries. New York, Garland. [Disponible en http://roa.rutgers.edu, núm. 234.]

BECKMAN, Jill N. \& Ringen, Catherine (2007). «Revisiting onset faithfulness constraints: evidence from Catalan voice neutralization». Trabajo presentado en el $15^{\text {th }}$ Manchester Phonology Meeting, Manchester.

(2008). «Coda devoicing: Does it exist?». Trabajo presentado en la $5^{\text {th }}$ OldWorld Conference in Phonology, Toulouse.

BenuA, Laura (1997). Transderivational identity: Phonological relations between words. Tesis doctoral. University of Massachusetts, Amherst.[Disponible en http://roa.rutgers.edu, núm. 259.] 
BERMÚDEZ-OTERO, Ricardo (2001).Voicing and continuancy in Catalan: a nonvacuous Duke-of-York gambit and a Richness-of-the-Base paradox. Manuscrito. University of Manchester. [Disponible en www.bermudez-otero.com.]

(2006). «Phonological domains and opacity effects: a new look at voicing and continuancy in Catalan». Trabajo presentado en el Workshop 'Approaches to phonological opacity', $29^{\text {th }}$ Generative Linguistics in the Old World Conference, Barcelona. [Resumen disponible en www.bermudez-otero.com.]

(2011). «Cyclicity». In: Marc van Oostendorp\& Colin Ewen\& Elizabeth Hume \& Keren Rice, eds., The Blackwell companion to phonology. Malden, MA, WileyBlackwell: IV, 2019-2048.

BertinetTO, Pier Marco (1999). «Boundary strength and linguistic ecology (Mostly exemplified on intervocalic /s/-voicing in Italian)». Folia Linguistica 33: 267-286.

BotмA, Bert (2011). «Sonorants». In: Marc van Oostendorp\& Colin Ewen\& Elizabeth Hume \& Keren Rice, eds., The Blackwell companion to phonology. Malden, MA, Wiley-Blackwell: I, 171-194.

BRADLEY, Travis G. (2005). «Sibilant voicing in Highland Ecuadorian Spanish». Lingua(gem) 2: 29-42.

Bradley, Travis G. \& Delforge, Ann Marie (2006). «Systemic contrast and the diachrony of Spanish sibilant voicing». In: Randall Gess \& Deborah Arteaga, eds., Historical Romance linguistics: Retrospectives and perspectives. Amsterdam, John Benjamins: 19-52.

CARRERA, Aitor (2011). L'occità. Gramàtica $i$ diccionari bàsics (occità referencial $i$ aranès). Lleida: Pagès editors.

CATAlÁn, Diego (1964). «El español en Canarias». In: Presente y futuro de la lengua española: Actas de la Asamblea de Filología del 1 Congreso de Instituciones Hispánicas. Madrid, Ediciones Cultura Hispánica: I, 239-280.

CAZACU, Boris\& Chiosa, Clara Georgeta\&CARAgIUMARIoțeAnU, Matilda \&GuȚU Romalo, Valeria \& BerCescu, Sorina (1967). Cours de langue roumaine. Bucuresţi, Editura Didactică şi Pedagogică.

ColinA, Sonia (2009). «Sibilant voicing in Ecuadorian Spanish». Studies in Hispanic and Lusophone Linguistics 2: 3-29.

Dalbera-Stefanaggi, Marie-José (1997). «Corsica». In: Martin Maiden \& Mair Parry, eds., The Dialects of Italy. London / New York, Routledge: 303-310.

DELL, François (1995). «Consonant clusters and phonological syllables in French». Lingua 95: 5-26.

DURAND, Jacques (1992). Fundamentos de fonología generativa y no lineal. Madrid, Teide.

DYCKSTRA, Gerald (1955). Spectrographic analysis of Spanish sibilants and its relation to Navarro's physiological phonetic description. Tesis doctoral. University of Michigan.

FreIXEIRO, Xosé Ramón (2006). Gramática da lingua galega. I. Fonética e fonoloxía. $2^{\mathrm{a}}$ ed. Vigo, A Nosa Terra. 
GianNELli, Luciano \& CRAVENS, Thomas D. (1997). «Consonantal weakening». In: Martin Maiden \& Mair Parry, eds., The Dialects of Italy. London / New York, Routledge: $32-40$.

GuitarT, Jorge M. (1980). «Aspectos del Consonantismo Habanero: Reexamen descriptivo». In: Gary E. Scavnicky, ed., Dialectología Hispanoamericana, estudios actuales. Washington, Georgetown University Press: 32-47.

HuALDE, José Ignacio (2011). «Sound change». In: Marc Van Oostendorp\& Colin J. Ewen\& Elizabeth Hume \& Keren Rice, eds., The Blackwell Companion to Phonology, vol. IV. Malden/Oxford, Wiley-Blackwell: 2214-2235.

(2013). «Phonological awareness and conventionalization in sound change».Trabajo presentado en Phonetics and Phonology in Iberia 2013, Lisboa.

HuALDE, José Ignacio \& NADEU, Marianna (2011). «Lenition and phonemic overlap in Rome Italian». Phonetica 68: 215-242.

HuALDE, José Ignacio\& Simonet, Miquel\& Nadeu, Marianna (2011). «Consonant lenition and phonological recategorization».Laboratory Phonology2.2: 301-329.

ITÔ, Junko\& Mester, Armin \& PADGETT, Jaye (1995). «Licensing and underspecification in Optimality Theory». Linguistic Inquiry 26: 571-613.

JANSEN, Wouter (2004). Laryngeal contrast and phonetic voicing: A laboratory phonology approach to English, Hungarian, and Dutch. Tesis doctoral. University of Groningen.

JIMÉNEZ, Jesús (1999). L'estructura sillàbica del català. València / Barcelona: IIFV / Publicacions de l'Abadia de Montserrat.

JIMÉNEZ, Jesús \& LLORET, Maria-Rosa (2008). «Asimetrías perceptivas y similitud articulatoria en la asimilación de sonoridad del catalán». Cuadernos de Lingüística del I.U.I. Ortega y Gasset 15: 71-90. [Disponible en http: //www.uv.es/foncat, \#22]

JONES, Michael (1988). «Sardinian». In: Martin Harris \& Nigel Vincent, eds., The Romance Languages. London / New York, Routledge: 314-350.

KIPARSKY, Paul (1985). «Some consequences of Lexical Phonology». Phonology Yearbook 2: 85-138.

KRÄMER, Martin (2009). The phonology of Italian. Oxford / New York, Oxford University Press.

LIPSKI, John (1989). «/s/-Voicing in Ecuadorian Spanish: Patterns and principles of consonantal modification». Lingua 79: 49-81.

(1990). «Aspects of Ecuadorian vowel reduction». Hispanic Linguistics 4.1: 119.

Lloret, Maria-Rosa\& PONS, Clàudia \& JiMÉNEZ, Jesús (2001). «Rara afis, rara avis. O del comportament fonològic especial de les fricatives labiodentals en català». In: Maria-Rosa Lloret \& Clàudia Pons, eds., Noves aproximacions a la fonologia a la morfologia del català. Volum d'homenatge a Max Wheeler. Alacant, Institut Interuniversitari de Filologia Valenciana: 353-403.

LOMBARDI, Linda (1995). Why Place and Voice are different: Constraint interactions and feature faithfulness in Optimality Theory. Manuscrito. University of Maryland, College Park. Publicado en 2001: «Why Place and Voice are different: Constraint specific alternations in Optimality Theory». In: Linda Lombardi, ed., Segmental 
Phonology in Optimality Theory. Cambridge, Cambridge University Press: 13-45. [Disponible en http://roa.rutgers.edu, \#105.]

(1996). «Restrictions on direction of voicing assimilation: an OT account». Maryland Working Papers in Linguistics 4: 89-115. [Disponible en http://roa.rutgers.edu, \#246.]

(1999). «Positional faithfulness and voicing assimilation in Optimality Theory». Natural Language and Linguistic Theory 17: 267-302.

LONCHAMP, François (2010). «La transcription phonétique du français». Versión 03 del 11/11/2010,disponible

http://francois.lonchamp.free.fr/TranscriptionPhonetique/transcriptionPhonetique.pd $\mathrm{f}$.

LOPORCARO, Michele (1995). Prosodic domains in Romance phonology. Manuscrito. University of Zürich.

(1999). «Teoria fonologica e ricerca empirica sull'italiano e i suoi dialetti».In: Paola BENINCÀ\& Laura VANELli \& Alberto MiOnI, eds., Fonologia e morfologia dell'italiano e dei dialetti d'Italia: atti del 31. Congresso della Società di linguistica italiana, Padova, 25-27 settembre 1997. Roma, Bulzoni: 117-151.

MADDIESON, Ian \&LADEFOGED, Peter (1996). The sounds of the world's languages. Oxford / Cambridge, Blackwell.

MALLINSON, Graham (1988). «Rumanian». In: Martin Harris \& Nigel Vincent, eds., The Romance Languages. London / New York, Routledge: 391-419.

Martín ButragueÑo, Pedro (en prensa). «Oclusivas orales: descripción de la variación». In: Juana Gil \& Joaquim Llisterri, eds., Fonética y fonología descriptivas de la lengua española. Madrid, Castalia / Consejo Superior de Investigaciones Científicas.

MASCARÓ, Joan (1987). «A reduction and spreading theory of voicing and other sound effects». Manuscrito. Universitat Autònoma de Barcelona, Bellaterra. Publicado en 1995: "A reduction and spreading theory of voicing and other sound effects». Catalan Working Papers in Linguistics 4: 267-328

MAteus, Maria Helena \& D'ANDRADE, Ernesto (2000).The Phonology of Portuguese. Oxford, Oxford University Press.

Noske, Roland (1993). A Theory of Syllabification and Segmental Alternation. With studies on the phonology of French, German, Tonkawa and Yawelmani. Tübingen, Niemeyer.

$N G L E=$ Real Academia Española \& Asociación de Academias de la Lengua Española (2011). Nueva gramática de la lengua española. Fonética y fonología. Madrid, Espasa.

OFTEDAL, Magne (1985).Lenition in Celtic and in insular Spanish: The secondary voicing of stops in Gran Canaria. Oslo, Universitetsforlaget.

Peyrollaz, Marguerite \&BARA DE TOVAR, Marie-Louise (1954). Manuel de phonétique et de diction françaises. Paris, Librairie Larousse.

Prince, Alan \& SMOLEnSKy, Paul (1993 [2004]). Optimality Theory. Constraint interaction in Generative Grammar. Malden/Oxford/Carlton, Blackwell. [Disponible en http://roa.rutgers.edu, \#537.] 
RICE, Keren (1993). «A re-examination of the feature [sonorant]: The status of 'sonorant obstruents'». Language 69: 308-344.

RICE, Keren \& AVERY, Peter (1989). «On the interaction between sonorancy and voicing». Toronto Working Papers in Linguistics 10: 65 - 82.

(1991). «On the relationship between laterality and coronality». In Carole PARADIS \& Jean-François PRUNET, eds., The special status of coronals: Internal and external evidence. San Diego, Academic Press: 101-124.

Rose, Sharon \& WALKER, Rachel (2004). «A typology of consonant agreement as correspondence». Language 80: 475-531.

STEN, Holger (1956). Manuel de phonétique française. København, Ejnar Munksgaard.

STRYCHARCZUK, Patrycja\& SIMON, Ellen (2013). «Obstruent voicing before sonorants. The case of West-Flemish». Natural Language and Linguistic Theory 31: 563-588.

StrycharczuK, Patrycja\& VAN'T Veer, Marijn\& Bruil, Martine \& LinKe, Kathrin (2013). «Phonetic evidence on phonology-morphosyntax interactions: Sibilant voicing in Quito Spanish». Jounal of Linguistics [FirstView article]: 1-50. [Disponible en CJO2013. doi:10.1017/S0022226713000157.]

TORREBLANCA, Máximo (1976). «La sonorización de las oclusivas sordas en el habla toledana». Boletín de la Real Academia Española 56: 117-145.

(1983). «Las sibilantes sonoras del oeste de España: ¿arcaísmo o innovaciones fonéticas?». Revista de Filología Románica 1: 61-108.

(1986). «La sonorización de /s/ y / $/$ / en el noroeste toledano». Lingüística Española Actual 8: 5-19.

WALKER, Douglas C. (1984). The Pronunciation of Canadian French. Ottawa,University of Ottawa Press.

WAlter, Henriette (1977). La phonologie du français. Paris, Presse Universitaires de France.

WETZELS, Leo W. \& MASCARÓ, Joan (2001). «The typology of voicing and devoicing». Language 77: 207-244.

WheELER, Max W. (1988). «Occitan». In: Martin Harris \& Nigel Vincent, eds., The Romance Languages. London / New York, Routledge: 246-278.

(2005). The phonology of Catalan. Oxford, Oxford University Press. 